\title{
Potentials of energy consumption measurements in office environments
}

\author{
Timo Jakobi \\ Workgroup on Human Computer Interaction \\ University of Siegen \\ Siegen, Germany \\ timo.jakobi@uni-siegen.de
}

\author{
Gunnar Stevens \\ Information Systems \\ Bonn-Rhein-Sieg University of Applied Science \\ Sankt Augustin, Germany \\ gunnar.stevens@uni-siegen.de
}

\begin{abstract}
Reducing energy consumption is one of the most pursued economic and ecological challenges con-cerning societies as a whole, individuals and organizations alike. At the same time, ubiquitous compu-ting provides expansive opportunities for measuring energy consumption. Still, few studies have touched on understanding what use could be made of the richness of data. This holds especially true for office environments so far, though they account for almost every second workplace in modern economics. In this paper, we present findings of an energy feedback study conducted in an organizational context using behavioral change oriented strategies to raise energy awareness. For an improvement of feedback based saving strategies, we conclude that energy feedback should fit to the local organiza-tional context to succeed and should consider typical work patterns to foster accountability of con-sumption. Next to demonstrating its potentials, we finally sketch how, based on our experiences, energy consumption data can be put to further use, for supporting sustainable action in organizations across departments.
\end{abstract}

Keywords-Eco-feedback; Sustainable Interaction Design; Workplace; Case-Study.

\section{INTRODUCTION}

Sustainable Interaction Design (SID) (Blevis, 2007) is a field of growing importance for HCI. Within the course of establishment, the HCI community evolved quickly and in an unbalanced way especially in the context of energy feedback, whereas SID has predominantly focused on the research of domestic energy use (Froehlich et al., 2010; Pierce et al., 2008; Schwartz et al., 2014; Van Dam et al., 2010). Within organizations however, common approaches to reducing energy costs are still artefact-oriented while behavior-based energy saving potentials are far less addressed (Foster et al., 2012; Schwartz et al., 2013a). Strategies for reducing the carbon footprint of organizations commonly stem from Green IT research (Melville, 2010; Murugesan, 2008) investigating concepts such as energy-efficient hardware and algorithms, green data center design, server virtualization and automated power management. Further technologically-oriented measures are IT-enabled energy-efficient building- and applianceautomation (Wong et al., 2005). For instance, smart solutions for HVAC and light control can help to reduce energy consumption without employees being disrupted in their routines.
In order to tap into the full saving potential, however, technological rearrangements should be supplemented by behavioral measures known from Sustainable Interaction Design research in domestic contexts (Blevis, 2007; DiSalvo et al., 2010). Still, as organizational contexts differ in many aspects from home environments, it is still an open research question how to adapt findings from the domestic setting to the workplace and how existing design concepts might need adaption.

Addressing this gap, we conducted a study, deploying smart sensors in an office environment to measure energy consumption at device level. We conducted a one-week information campaign and measured follow-up effects in an office environment. Additionally, we tested different approaches of personalized feedback reporting, studied its effects on the individual and overall consumption and explored opportunities to embed information on energy consumption into organizations, in order to enable further use of this data. In this paper we demonstrate the potentials of energy feedback in the workplace and shed light on future possibilities and challenges, such as the integration into BPM cycles and tailoring feedback to work practices in organizations.

\section{BEHAVIORAL ENERGY SAVING IN THE OFFICE}

\section{A. Behavioral Environmental Research}

Behavioral environmental research first got a boost during the oil crisis of the 1970's (Poortinga et al., 2003). Around that time, research by ecologic psychologists started to take an interest in the influence of behavior on energy consumption (Stern and Aronson, 1984) and investigated consumption feedback as a means of encouraging energy conservation (Brandon and Lewis, 1999). At the same time, a body of theoretical approaches emerged within environmental research, seeking to understand individuals' (un-)sustainable behaviors (Jackson, 2005).

One of the most influential approaches has its roots in rational choice theory (Jackson, 2005), arguing that energy-relevant behavior is steered by the active and informed decisionmaking of consumers, who weigh pros and cons such as the satisfaction of needs with costs and thus act according to their judgment. Other theories (like Stern's Value Belief Norm Theory (Stern, 2000)) also consider subjective norms, beliefs, 
and the influence of social surroundings. By and large, such concepts of norms and rational behavior are the theoretical foundation of persuasion and feedback campaigns (Froehlich et al., 2010). In particular, design concepts such as providing direct feedback, enabling social comparison and supporting goal setting were inspired by these theories (Abrahamse et al., 2005).

\section{B. Designing for Behavioral Change}

Technological innovations have opened new opportunities to promote pro-environmental behavior and pushed novel solutions into the market (Darby, 2006). In particular, Sustainable Interaction Design (SID) has become an established subfield of HCI, challenging the immateriality of energy (Pierce and Paulos, 2010) and exploring how consumption could be made visible and meaningful (Brandon and Lewis, 1999).

The spectrum of feedback design has become very wide, reaching from artistic solutions like the Power Aware Cord (Gustafsson and Gyllenswärd, 2005), over pragmatic ones like Watt-Lite [31], social norm oriented approaches (Foster et al., 2010) to HEMS integrating multiple features into a homeoriented system of services (Schwartz et al., 2013a). In addition, several empirical studies also demonstrate the effectiveness of eco-feedback in not only raising awareness but empowering consumers to implement savings as well (Darby, 2006; Van Dam et al., 2010). Despite this, feedback systems are mainly studied in the domestic context.

\section{Energy Conservation for Office Workers}

In terms of designing interventions for organizational settings, there are only a few guidelines relating to the design and conducting of behavioral change oriented eco-campaigns.

Matthies et al. (Matthies and Wagner, 2011) for instance have developed a tailored program including checklists, HOW-TOs, sample templates etc. to conduct eco-campaigns in public administration buildings. The authors mainly focus on classic materials like posters, flyers, information brochures and letters from superior authorities. They also give advice on how to incorporate emails and websites, but suggestions for using smart technologies have yet to be integrated in these toolboxes. Azar \& Menassa (Azar and Menassa, 2012) have investigated the design of organizational eco-feedback. They developed a decision support framework for conducting energy saving measures in commercial buildings. Initially, general design guidelines and wireframe sketches were developed by Foster et al. (Foster et al., 2012) using focus group sessions. Based on a literature review about techniques of intervention appropriate for the workplace, Yun (Yun et al., 2013) implemented a first functional prototype of an energy-dashboard.

The few studies evaluating eco-feedback in organizational contexts show mixed results. Carrico and Riemer (Carrico and Riemer, 2011) show that providing monthly feedback with a motivating message has a positive effect on the energy saving behavior of university employees. Installing eco-feedback applications on the desktop of university employees, Murtagh et al. (Murtagh et al., 2013) also found a significant reduction of consumption. However, they noticed a complex relationship between feedback and behavior and found manifold reasons 'not to switch things off'. Using smart metering technology in a research institute, Schwartz et al. (Schwartz et al., 2010) observed significant positive effects too, but only short-term, with conservation fading successively over time.

Another issue addresses the question, which intervention strategies could and should be applied, in organizational contexts. For instance, in the domestic setting comparative feedback is a well-established strategy (Froehlich et al., 2010). Yet, as we will show below, this strategy cannot be transferred fully as it raises conflicts with privacy issues as well as values and norms of local organizational culture. This issue has already been indicated by Schwartz et al. (Schwartz et al., 2010), who noted that some employees interviewed are quite guarded in showing their personal consumption to colleagues or other parts of the organization, because they were afraid of misinterpretation or misuse.

\section{METHOD}

In order to explore the potentials of behavioral energy saving measures, we investigated the effects of information and feedback campaigns in a company which can be characterized as follows:

The company is a financial service provider with over 30 branch offices and headquarters including a service center and two administrational buildings. The company's culture is coined by high seriousness and solidity. As another characteristic, the company is firmly rooted regionally and shows strong colleagueship.

For the study, we chose a department within the administration, mainly engaged in construction financing and architecture and which consists of 30 workplaces. Furthermore, there is a recreational room, including a fully equipped kitchen and a printing pool with four printers, a plotter, a scanner and a plan copier. With offices accommodating up to three employees, we equipped every workplace in the department with the measurement system to include it in our study. Standard equipment includes a cellular phone and base station. Almost everyone uses a personal computer and one flat screen monitor, with 18 participants having a regular PC and nine employees

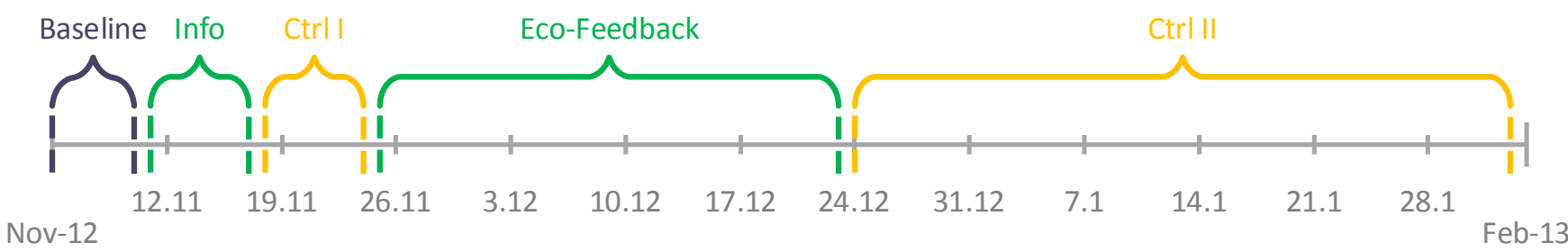

Figure 1: Timeline of interventions and measurements throughout the project 
working at virtual desktops. A few employees were provided with a second screen for special purposes and three participants had two computers each, constituting the department's IT. Private appliances are not used regularly. Appliances in pooled and recreational areas were captured, too, while we excluded light and HVAC, as they were managed fully automatically.

\section{A. Intervention Design and Realization}

We split our intervention into different stages (cf. Figure 1) including a baseline measurement, a one-week informationcampaign and two controlling measurements plus feedback reporting.

In the first phase, we conducted a two-week baseline measurement. Secondly, we mounted a one-week ecocampaign, which we adjusted to the company's specific conditions. During the third phase we measured the effects after campaigning over a period of two weeks. Furthermore, we had the opportunity to provide additional personalized, weekly feedback reports for the next three weeks. Afterwards, we kept the sensors in place for seven more weeks until February to measure follow-up effects.

\section{1) Baseline}

The goal of our baseline measurement was to provide statusquo data, therefore allowing comparison of changes in energy consumption in later phases with default values at appliance level.

The staff association was informed about the scope of the campaign, the installation of sensors and the handling of sensitive data. After consulting the management, it became apparent that some privacy concerns needed to be addressed, which is why we had one of the organization's employees create pseudonyms before any data was sent to us for processing. Additionally, we informed the management about our project, in order to comply with security regulations such as fire protection. Aiming for a baseline that would be as close as possible to normal usage, we avoided any unnecessary announcements. We were however asked to write an unobtrusive Email and we also introduced our study in regular fixed meeting. Additionally, sensors were sometimes visible to participants.

\section{2) Information and motivation campaign}

The aim of the campaign was to increase employees' awareness of energy-saving potential at the workplace as well as motivating employees to contribute and communicate information on opportunities to save energy. At a conceptual level, we followed examples from literature (Carrico and Riemer, 2011; Kastner and Matthies, 2013; Matthies and Wagner, 2011). Yet it became evident that interventions and materials had to be adjusted depending on the particular organizational context and in order to respect concerns both of management and employees regarding the organization's culture and their individual right to, and concerns about, privacy.

Additionally, the campaign had to be aligned with the organizational values and norms, in this case especially its culture of seriousness and regional rootedness. Experimental aspects and English terms on information material were relinquished and German wording was used instead. As another type of incentive, we proposed using a lottery or motivating employees, which the management perceived to be inappropriate. The preferred solution focused on motivating via hierarchical communication. Furthermore, since paperbased information distribution is widely established, we fostered the use of media such as posters, postcards, stand-up displays and hand-outs. Interactive media were seldom used, focusing on spreading information rather than motivating directly with activities changing every day.

As a core of measures for raising awareness, the information campaign also featured the presence of an energy consumption expert at an information desk placed centrally in the department and open for all kinds of questions regarding energy generally. This information desk was positioned in the foyer of the main building foyer and was exposed to heavy traffic, especially around lunch breaks.

3) Individual Design of Feedback Solutions

As a main benefit of smart metering technology, it allows consumers to receive feedback on individual consumption patterns in real-time. Making use of this advancement, we deployed high granular digital metering infrastructure in order to implement a comprehensive smart energy management system for employees, covering the whole process from data gathering to processing and delivery. As there was no commercial system available that fulfilled all requirements, we developed an individualized reporting solution for personal feedback. In addition to individual feedback sheets, we created an anonymous bulletin report of aggregated consumption for the whole department. This report was hung out on the

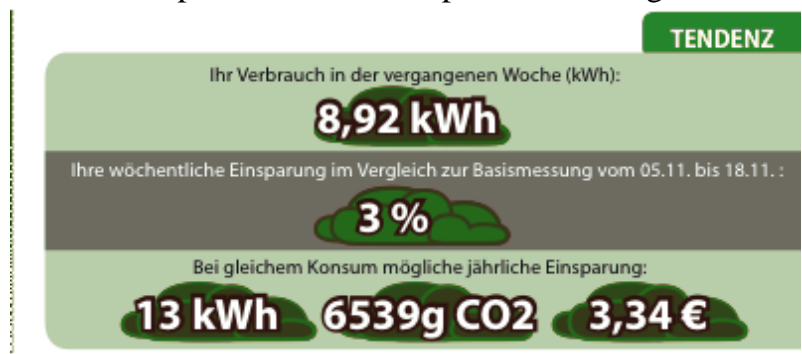

\section{WETTERINFO}

Die folgende Grafik zeigt Ihnen Ihren Energieverbrauch in der letzten Woche im Vergleich zur Basismessung vom 05.11. bis zum 18.11. an den jeweiligen Tagen. Je besser das Wetter, desto mehr Energie haben Sie gegenüber der Basismessung einsparen können.

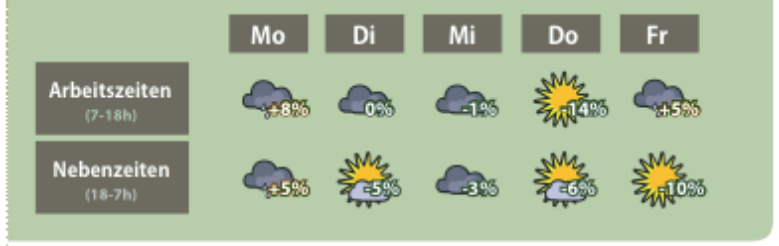

VERGLEICH ARBEITS- UND FREIZEIT

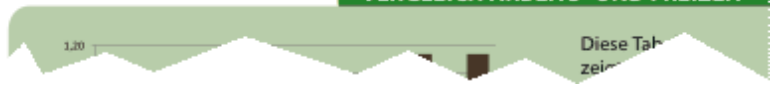

Figure 2: Part of a personalized feedback sheet distributed to workers featuring overall consumption, comparison to last week, different reference systems and a weather metaphor 
billboard and updated weekly.

Our design followed a pragmatic participatory-oriented approach, where we took up design ideas from literature (Froehlich et al., 2010; Schwartz et al., 2013b) and discussed them with employees and management in informal talks as well as in formal meetings. This input provided us with further ideas on how to improve our design and what kind of information was important for them. The aim was to transfer central information such as total consumption of the past week in kilowatt-hours, and percentage difference to previous week. Additionally, we included means for comparison among weekly consumption.

When discussing, how to provide meaningful information, it also became evident that wasting energy outside office hours is an important and yet neglected topic in eco-feedback research: Outside working hours, energy consumption at the workplace should be avoided entirely. Based on the characteristics of workplace energy consumption patterns, feedback systems should reflect this distinction (Masoso and Grobler, 2010; Murtagh et al., 2013; Wong et al., 2005). A second chapter therefore featured more disaggregated information, divided into working and non-working hours.

Although suggested in literature, comparative elements for feedback provision were considered as inappropriate within the organization. When we introduced the idea of showing a leaderboard within the weekly reports, this was perceived as possibly harmful for the collegial atmosphere which was valued to be one of the key characteristics for a good work environment for the department

Picking up on recommendations to provide a historic comparison, we additionally included a comparison between this week's daily consumption (Abrahamse et al., 2005; Froehlich et al., 2010; Schwartz et al., 2013a).

Based on the load-profiles gathered during the baseline period and talks with management, we broadly distinguished between 7 am - $6 \mathrm{pm}$ as working hours, $6 \mathrm{pm}$ to 7 am as nonproductive times and weekends. In future, we also plan to take public holidays into account. For this study, we used a timespan without national holidays included in our measurements.

The sheet was created based on data gathered in a semiautomatic fashion. For privacy reasons, records were neither passed on to colleagues nor were they accessible to superiors at any time. Pseudonymization meant that only one employee within Beta was in a position to allocate reports to each participant.

\section{RESULTS}

\section{A. Baseline Consumption}

Figure 2 presents the average weekly consumption of an office worker during the two weeks of baseline determination.

In the two weeks of baseline period, the 30 workplaces consumed $268.3 \mathrm{kWh}$ in total, with collective appliances amounting to $27.8 \mathrm{kWh}$ and the recreational room consuming $12.4 \mathrm{kWh}$.

Further load profile analysis showed that non-working hours and the weekend account for $37.2 \%$ (working hours: $3.2 \mathrm{kWh}$; non-productive hours: $1.2 \mathrm{kWh}$; weekends: $0.8 \mathrm{kWh}$ ). Employees as well as management were quite surprised about this finding, since working hours had already been subjected to a conservative estimation. Since the company had a strict firewall policy for security reasons which prohibited any remote access, there was no apparent reason for consumption outside working hours at all (on the upside, our finding revealed high energy saving potentials, to be tapped into simply by motivating workers to shut down appliances at closing-time).

\section{B. Effects of the Intervention on Consumption}

Figure 4 shows the course of energy consumption over the weeks of our study.

In the first week after the campaign, savings were mainly achieved outside working hours. The department's consumption was reduced by $13.3 \%$, with savings during working hours $(-7.5 \%)$ turning out lower than the significant changes at non-productive times $(-20.8 \%)$ and on weekends ($26.7 \%)$. At device level, reductions were not only found at the workplace $(-9.5 \%)$, but pooled consumption especially was significantly reduced by $56.2 \%$. This was mainly achieved by unplugging a large printer which was seldom used. Among recreational devices, usage stagnated $(+0.6 \%)$.

During the time of the subsequent personalized feedback provision from week four to week seven, average energy consumption was $27.1 \%$ lower than in the baseline period (working hours: -19.9\%; non-productive hours: $-37.2 \%$;
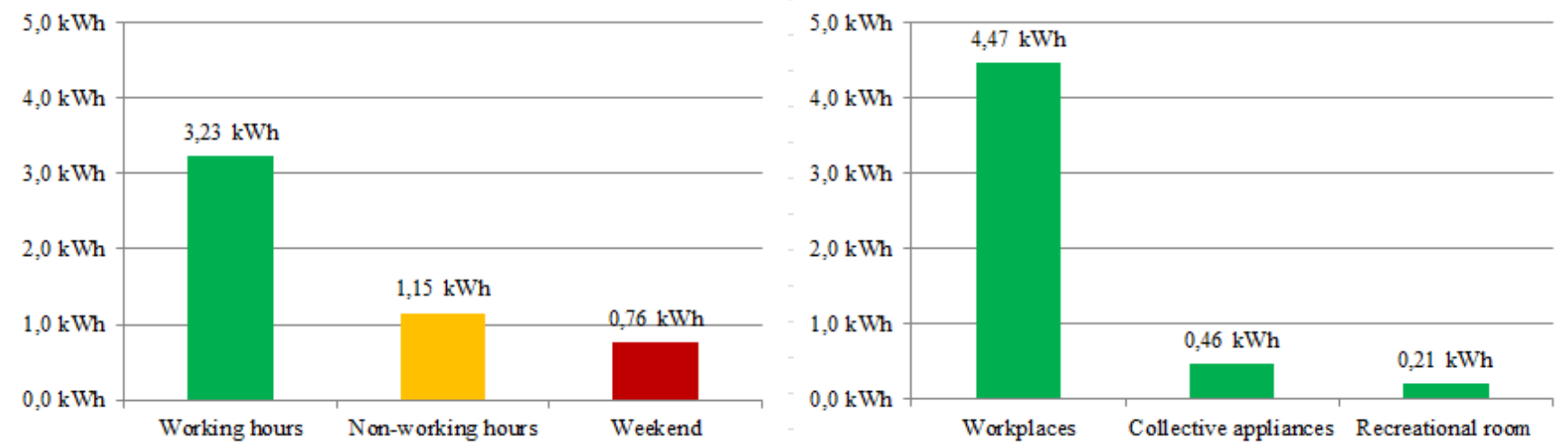

Figure 3: Energy consumption during Baseline period disaggregated 


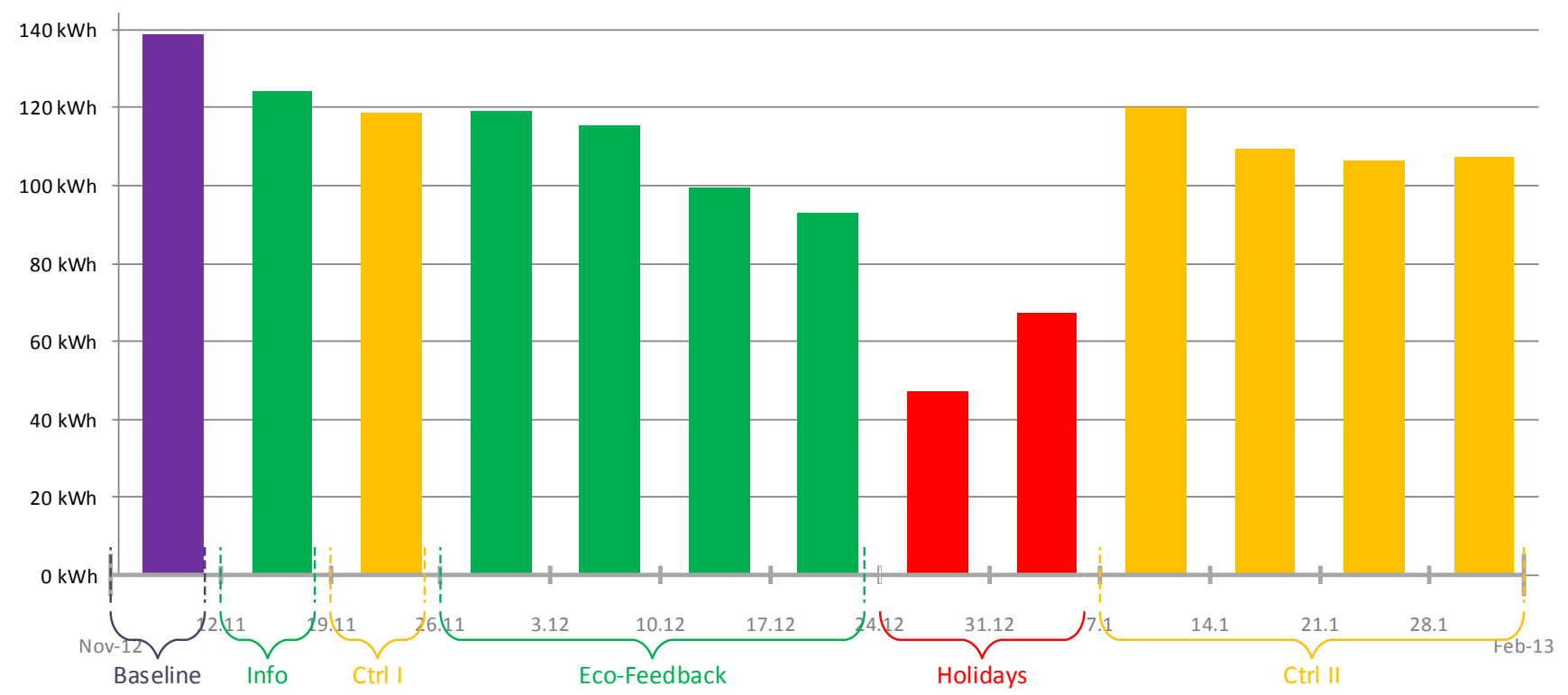

Figure 4: Total Energy consumption by week over the course of the project measured

weekends: -42.6\%). When the management was asked for feedback regarding any possible blind spots of our analysis, neither holiday plans nor illness reports showed any anomalies. Quite the contrary, it was pointed out that before the holidays the workload usually picks up and thus employees have to work more than regularly.

In order to study follow-up behavior, we excluded the weeks between Christmas and New Year's because of holidays. Instead, we calculated the average consumption from January $7^{\text {th }}$ until February 3rd (seven weeks after the last feedback report). In this period, total savings dropped, but even so $12.2 \%$ was conserved compared to baseline period. This was mainly archived outside working hours (non-productive hours: $-27.0 \%$; weekends: $-29.1 \%$ ). A small reduction of $3 \%$ was perceived in working hours between 7 am and 6 pm. Overall, the drop in conservation indicates that after the holiday break some old "bad" habits crept back into the participants' everyday lives.

\section{Empowerment and limitations}

With constant and close contact to the organizations' management and open talks with five employees at the beginning of the second control phase, we succeeding in gaining deeper insights into the employees' perception of the intervention. In our interviews we asked about the changes in their behavior, measures taken to save energy and their ideas for improving eco-campaigning in future ${ }^{1}$. While we found aspects of empowerment by providing individual feedback sheets to employees, we also found how obstacles prevented them from acting sustainably in several cases.

First, our consumption feedback provided basic awareness on energy conservation and was perceived in a positive way.

1 At the employees' request, these interviews were not recorded. After the meetings we immediately wrote down mind minutes of the interviews and joined them with the notes we took during the interviews
Information about the devices' consumption which had formerly been unknown and was therefore deemed surprising was evaluated.

P1:,I also liked that we could see that our thin clients - I mean, of course we know it, but to actually see that they consume much less [than fat clients]. And then, seeing that switching off the monitor makes such a difference... This should be the aim: To switch them off automatically or get people to do it on a regular basis."

Furthermore, on several occasions we found that the feedback provided enabled employees to make one-time infrastructural changes: For example, by reviewing the shared appliances, employees easily identified a large offset printer to be constantly hogging energy although it was reportedly only used once every other month.

Another typical action - especially pursued by the department's IT - was to try and find acceptable ways of saving energy when leaving the desk. This was especially tricky, as clients always had to log on to a server session for security reasons.

P1: „The way the settings are right now, my [computer] is operational in next to no time. That's effort worth making. This works!"

At the information desk, a discussion started about how to make usable trade-offs between saving energy and getting work done without being restricted by sustainability:

P2: „The [settings you made for the case you leave] for five minutes. I have adopted your settings for this now. Because otherwise, you keep forgetting it and using this, logically, you can't forget anymore."

As a third dimension, we found that people adopted more sustainable behavior, such as switching off their monitors during breaks and shutting down their Computers when leaving in the evening. On the other hand, it became obvious that both employees and management had doubts about long term effects on behavior resulting from a relatively short 
campaign. Strategies for long term effectiveness were therefore discussed:

P1:, The first few days were massive, people thought about it [saving energy] all the time, but it drops off. I can see it from my own behavior..."

P2: „There are certain things you can do with very small or almost no effort, you realize. The hard thing then is to always see how much you invest into more or less regular activities for raising awareness. "

Reflecting the "lessons learned" and including a discussion about options for improving eco-interventions in the future, people were curious about more intelligent ways to distinguish between wasted energy and energy consumption necessary for work:

P2: "Actually, you would have to compare working hours. Only then can you tell whether you saved energy during working hours or not. If I keep saving during working hours, but I work longer hours, where's the use in that? Then, after all, it [the consumption] has still increased. [...] But you'd probably only be able to check that with time sheets."

While impractical, it was also clear that the culture of the organization would not allow for the introduction of such sheets. Solutions discussed about automatically detecting working states by energy consumption were rejected as employees told us that they sometimes work without the computer or outside the office, too.

\section{INTEGRATION INTO ORGANIZATIONAL STRUCTURES}

In summary, the study demonstrates that behaviorally-oriented measures applied in domestic contexts (Abrahamse et al., 2005; Darby, 2006; Froehlich et al., 2010; Van Dam et al., 2010) generally prove to be promising in the office setting, too and is thus in line with other studies(Carrico and Riemer, 2011; Schwartz et al., 2010). Eco-feedback design for the workplace is quite a novel topic in HCI. Hence it is honorable to uncover common requirements and identify canonical design issues (Azar and Menassa, 2012; Foster et al., 2012; Yun et al., 2013). Yet, as noted by He et al. (He et al., 2010) one size does not fit all. While the original remark refers to people's diverse motivation, our study shows that it also holds true with regard to heterogeneity of organizational settings.

We showed that our sustainability efforts were successful, but remained a one-time intervention with limitations concerning process and structural sustainability, intelligence of the algorithms used and long-term effects.

In the following we discuss some of these topics in more detail, helping to transfer existing concepts and findings to organizational context.

\section{A. Designing interventions depends on the culture of the organization}

During collective planning workshops, we found that the organizations' self-image had a strong impact on the design of our interventions, too. While competitive persuasive approaches (like social comparison or goal setting) have proven successful in many cases (as shown in domestic settings) (Abrahamse et al., 2005; Foster et al., 2010; Froehlich et al., 2010), any kind of competition within the campaign (e.g. gamification) was perceived as inappropriate by the management. In particular, we recommended a leaderboard where office workers could compare their energy data against peers in the weekly reports. This was refused, since competitive elements would not fit into the general culture and self-image of the company. Therefore the design form, style, and use of emotions had to be adapted to the particular organizational corporate design, existing communication channels, technical infrastructure and interests of the user group, as certain elements were considered to not fit into the collegial culture by management. This reveals that in addition to competitively oriented approaches, collaborative approaches for energy saving should be investigated in the future.

The design of our information material was received positively, but nevertheless there is still potential for optimization. For instance, the personalized weekly reports were considered helpful as a direct feedback mechanism on individual behavior. In the context of financial business, employees emphasized the usefulness of money as the major feedback unit. On the other hand, information on energy efficiency in domestic settings, despite being valued generally, was perceived as too broad and untailored to individual living conditions.

\section{B. Privacy}

Another point of importance was the topic of privacy: Even before our study started, both management and workers made it very clear that energy consumption data was perceived to be sensitive. It was therefore important that (a) no data would be stored on external servers other than the universities' and (b) the anonymity of employees would be guaranteed in such a way that neither management nor colleagues were able to match consumption patterns to specific employees.

For instance, our data revealed that all employees, with only very small deviations, show up around 8 am and leave again around $5 \mathrm{pm}$. These business hours are not mandatory, since employees have flexible schedules. Comparing such data with public appointment calendars however could provide the basis for close monitoring of working hours. Furthermore, the individualized digital metering data was perceived as a private good and the right of access should be granted only to the corresponding employee (see also (Schwartz et al., 2010) for a similar observation). We therefore abandoned comparative elements in this study.

As described, we were unable to use smart plugs as an off-theshelf reporting tool as they were not customizable in terms of taking cultural requirements, privacy and access control of the participating organizations into account.

\section{Adapting to work practices}

In accordance with other studies (Masoso and Grobler, 2010; Murtagh et al., 2013; Webber et al., 2006), our findings show significant consumption to occur outside of working hours 
(37.1\%) and leaving the office is probably the single most critical event for decisions on wasting or saving energy. Although a well-known fact, it seems we are the first to address this consideration in eco-feedback design by distinguishing between working and non-working hours. The savings achieved as well as the positive comments of our participants compel us to argue that giving the right feedback and making wastage accountable can provide a great benefit.

In detail, however, our user's comments also show that our algorithmic approach was too straightforward. In particular, it does not consider events such as appointments outside the office or employees doing overtime. Therefore instead of earning special praise, our report showed a rain cloud as feedback, since the employee working extra hours consumed more energy than normal. This was judged to be misleading and unfair.

An essential topic for the future is therefore to develop more sophisticated methods for more precise analysis and feedback provision. Campaigns could benefit, for example, from a better understanding of systematic behavioral change and its connected effects. Moreover, our study shows that when consumption is declared to be waste, this is highly dependent on context and the local actors' notion of the consumed services (Schwartz et al., 2013b; Strengers, 2011). Additionally, accounting for and compensating the sources of irritation of specific special events (e.g. holidays, illness and appointments away from the office) will be necessary for more accurate feedback. Hence, future analytic algorithms and concepts for visualization of energy data need further refinement which should constantly be evaluated in terms of fitting to organizational and user practice. This especially holds true for the open question of how to appropriately prorate consumption of shared appliances to individual energy consumption.

\section{Making conservation sustainable}

In the aftermath of our second control measurements, we observed energy conservation to be declining after a while. As the context remained largely unchanged at this time, it seems that employees started to fall gradually but progressively back into old "bad" habits. A similar phenomenon is noted by Blevis (Blevis, 2007), while Azar and Menassa (Azar and Menassa, 2012) provide a counter example. This shows a further challenge to sustaining behavioral changes when designing smarter feedback solutions. Regarding this, Corradi et al. (Corradi et al., 2012) propose reducing the cognitive demands of energy saving behaviors. In addition, we propose that energy saving should become part of the routinized, socio-material practices (Reckwitz, 2002). A promising yet under-investigated approach in this direction presents the fusion of both technological and behavioral-oriented concepts. In particular, getting the user in the loop of automated power management could help to increase their usability and user acceptance. Complementary, smart eco-assistant systems could help to relieve people from tedious sustainability work like checking that all devices are turned off at closing time. With the absent of monetary motivation, it will furthermore be interesting to gain a better understanding of how to motivate employees for long term sustainable behavior and innovativeness at the workplace, as Krüp et al. have contributed recently (Krüp et al., 2014).

On several occasions, we observed how employees had ideas for increasing energy efficiency, e.g. by changing configurations, work structures, and processes. However, within our study, they were largely unable to overcome such barriers and, as a consequence, abandoned their ideas. Management, being the single accountable site for introducing new processes, was sometimes unaware and at other times failed to support the new ideas which meant the workers' local expertise could not be put into effect. While at some point this might have been for good reasons, such as IT-security within the financial service provider, some ideas just did not make it to the ears of the decision makers. This highlights research questions which remain open, such as ways of introducing workers to process restructuring as promoted by Nolte(Herrmann and Nolte, 2010). A further question regards ways and challenges of introducing measured sustainability data into ways of improving an organizations sustainability, in order to allow this information to be referred to for decision making (Jakobi et al., 2014).

\section{LIMITATIONS}

Due to the small sample size and the relatively short measurement period, our study could indeed give a first impression, but could not claim any statistical representativeness. In addition, Figure 3 shows that not only campaigning itself, but also even announcing an intervention and installing sensors had positive effects on energy behavior among participants. This is why the actual baseline would be somewhat higher than the one we captured. Thus savings should be rated slightly higher as well.

Another general limitation is that (to the best of our knowledge) there is no generally accepted procedure for dealing with seasonal effects, holidays, extra hours etc. Handling such events consistently is necessary in order to estimate the effects of behavioral change transparently and comparably and to separate them from perturbations. Murtagh et al. [23], for instance, did not consider them at all to avoid distortion of the measured data, thus bearing the risk of drawing false conclusions (e.g. stating a behavioral change, although it was mainly a vacation effect).

In our study, we checked that our data is consistent in terms of numbers of employees taking holidays or being off sick. In addition we treated official holidays as non-working days and used mean consumption of the four-day-week instead. We had no apparent holidays, but we excluded the vacation time around Christmas and New Year`s from our calculation.

\section{CONCLUSION}

Sustainable HCI, so far, has mainly focused on the domestic context. However, our study demonstrates that it is worth investigating in behavior-based interventions at the workplace. As our results show, the consumption could be reduced between $5 \%$ and $12 \%$ in both cases just by behavioral change. It also reveals that management support, compliance with 
privacy, security demands, and respecting the organizational context are important factors of acceptance of both organizations and employees as well.

Due to the small number of empirical studies on sustainable HCI in the work context, however, we want to validate our results in further studies, in order to better understand demands in terms of privacy, integration of consumption data into organizational strategic planning and more intelligent algorithms.

\section{REFERENCES}

[1] Abrahamse, W., Steg, L., Vlek, C., and Rothengatter, T. A review of intervention studies aimed at household energy conservation. Journal of Environmental Psychology 25, 3 (2005), 273-291.

[2] Azar, E. and Menassa, C.C. A comprehensive analysis of the impact of occupancy parameters in energy simulation of office buildings. Energy and Buildings 55, (2012), 841-853.

[3] Blevis, E. Sustainable interaction design: invention \& disposal, renewal \& reuse. Proceedings of the SIGCHI conference on Human ..., (2007), 503-512.

[4] Brandon, G. and Lewis, A. Reducing household energy consumption: a qualitative and quantitative field study. Journal of Environmental Psychology 19, 1 (1999), 75-85.

[5] Carrico, A.R. and Riemer, M. Motivating energy conservation in the workplace: An evaluation of the use of group-level feedback and peer education. Journal of Environmental Psychology 31, 1 (2011), 1-13.

[6] Corradi, N., Priftis, K., Jacucci, G., and Gamberini, L. Oops, I forgot the light on! The cognitive mechanisms supporting the execution of energy saving behaviors. Journal of Economic Psychology, (2012).

[7] 7. Van Dam, S.S., Bakker, C.A., and Van Hal, J.D.M. Home energy monitors: impact over the medium-term. Building Research \& Information 38, 5 (2010), 458-469.

[8] Darby, S. The effectiveness of feedback on energy consumption. A Review for DEFRA of the Literature on Metering, Billing and Direct Displays. Environmental Change Institute, University of Oxford, Oxford, 2006.

[9] DiSalvo, C., Sengers, P., and Brynjarsdóttir, H. Mapping the landscape of sustainable HCI. Proceedings of the 28th international conference on Human factors in computing systems - CHI '10, (2010), 1975.

[10] Foster, D., Lawson, S., Blythe, M., and Cairns, P. Wattsup?: motivating reductions in domestic energy consumption using social networks. Proceedings of the 6th Nordic Conference on Human-Computer Interaction: Extending Boundaries, ACM (2010), 178-187.

[11] Foster, D., Lawson, S., Wardman, J., Blythe, M., and Linehan, C. Watts in it for me?: design implications for implementing effective energy interventions in organisations. Proceedings of the 2012 ACM annual conference on Human Factors in Computing Systems, ACM (2012), 2357-2366.

[12] Froehlich, J., Findlater, L., and Landay, J. The design of eco-feedback technology. Proceedings of the 28th international conference on Human factors in computing systems - CHI '10, ACM Press (2010), 1999-2008.

[13] Gustafsson, A. and Gyllenswärd, M. The power-aware cord: energy awareness through ambient information display. CHI'05 extended abstracts on Human factors in computing systems, ACM (2005), 14231426.

[14] He, H.A., Greenberg, S., and Huang, E.M. One size does not fit all: applying the transtheoretical model to energy feedback technology design. Proceedings of the 28th international conference on Human factors in computing systems, ACM (2010), 927-936.

[15] Herrmann, T. and Nolte, A. The Integration of Collaborative Process Modeling and Electronic Brainstorming in Co-located Meetings. In G. Kolfschoten, T. Herrmann and S. Lukosch, eds., Collaboration and Technology. Springer Berlin Heidelberg, 2010, 145-160.

[16] Jackson, T. Motivating sustainable consumption. A review of evidence on consumer behaviour and behavioural change. A report to the
Sustainable Development Research Network, Surrey: Centre for Environmental Strategies, January (2005).

[17] Jakobi, T., Castelli, N., Nolte, A., Stevens, G., and Schönau, N. Towards Collaborative Green Business Process Management. (2014).

[18] Kastner, I. and Matthies, E. Implementing web-based interventions to promote energy efficient behavior at organizations - a multi-level challenge. Journal of Cleaner Production, (2013).

[19] Krüp, H., Kranz, J., and Kolbe, L. It's not for the money; it's the motives-The mediating role of endogenous motivations on IT employees' entrepreneurial behavior. (2014).

[20] Masoso, O.T. and Grobler, L.J. The dark side of occupants' behaviour on building energy use. Energy and Buildings 42, 2 (2010), 173-177.

[21] Matthies and Wagner. Change-Veränderung nachhaltigkeitsrelevanter Routinen in Organisationen. LIT Verlag Münster, 2011.

[22] Melville, N.P. Information systems innovation for environmental sustainability. MIS Quarterly 34, 1 (2010), 1-21.

[23] Murtagh, N., Nati, M., Headley, W.R., et al. Individual energy use and feedback in an office setting: A field trial. Energy Policy, 62 (2013), 717-728.

[24] Murugesan, S. Harnessing green IT: Principles and practices. IT professional 10, 1 (2008), 24-33.

[25] Pierce, J., Odom, W., and Blevis, E. Energy aware dwelling: a critical survey of interaction design for eco-visualizations. ... Interaction: Designing for Habitus and ..., (2008).

[26] Pierce, J. and Paulos, E. Materializing energy. Proceedings of the 8th ACM Conference on Designing Interactive Systems, (2010), 113.

[27] Poortinga, W., Steg, L., Vlek, C., and Wiersma, G. Household preferences for energy-saving measures: A conjoint analysis. Journal of Economic Psychology 24, 1 (2003), 49-64.

[28] Reckwitz, A. Toward a Theory of Social Practices: A Development in Culturalist Theorizing. European journal of social theory 2002, 2 (2002), 245-265.

[29] Schwartz, T., Betz, M., Ramirez, L., and Stevens, G. Sustainable energy practices at work. Proceedings of the 6th Nordic Conference on HumanComputer Interaction Extending Boundaries - NordiCHI '10, ACM Press (2010), 452-462.

[30] Schwartz, T., Denef, S., Stevens, G., Ramirez, L., and Wulf, V. Cultivating energy literacy: results from a longitudinal living lab study of a home energy management system. Proceedings of the SIGCHI Conference on Human Factors in Computing Systems, (2013), 11931202.

[31] Schwartz, T., Stevens, G., Jakobi, T., et al. What People Do with Consumption Feedback: A Long-Term Living Lab Study of a Home Energy Management System. Interacting with Computers 26, 3 (2014), iwu009.

[32] Schwartz, T., Stevens, G., Ramirez, L., and Wulf, V. Uncovering practices of making energy consumption accountable. ACM Transactions on Computer-Human Interaction 20, 2 (2013), 1-30.

[33] Stern, P.C. New Environmental Theories: Toward a Coherent Theory of Environmentally Significant Behavior. Journal of Social Issues 56, 3 (2000), 407-424.

[34] Stern, P.C. and Aronson, E. Energy Use: The Human Dimension. W.H. Freeman \& Company, 1984

[35] Strengers, Y.A. Designing eco-feedback systems for everyday life. Proceedings of the SIGCHI Conference on Human Factors in Computing Systems, ACM (2011), 2135-2144.

[36] Webber, C.A., Roberson, J.A., McWhinney, M.C., Brown, R.E., Pinckard, M.J., and Busch, J.F. After-hours power status of office equipment in the USA. Energy 31, 14 (2006), 2823-2838.

[37] Wong, J.K.W., Li, H., and Wang, S.W. Intelligent building research: a review. Automation in Construction 14, 1 (2005), 143-159.

[38] Yun, R., Scupelli, P., Aziz, A., and Loftness, V. Sustainability in the workplace: nine intervention techniques for behavior change. In Persuasive Technology. Springer, 2013, 253-265. 